\title{
ATTENUATED INFECTIOUS HEMATOPOIETIC NECROSIS VIRUS (IHNV) WITH REARRANGED GENE ORDER AS POTENTIAL VACCINE
}

\author{
Ronan N. Rouxel ${ }^{1}$, Carolina Tafalla ${ }^{1,2}$, Emilie Mérour ${ }^{1}$, Esther Leal ${ }^{2}$, Stéphane \\ Biacchesi $^{1 \$}$, Michel Bremont ${ }^{1 *}$ \\ ${ }^{1}$ VIM, INRA, Université Paris-Saclay, 78350, Jouy-en-Josas, France, ${ }^{2}$ Centro de \\ Investigacion en Sanidad Animal (CISA-INIA), Valdeolmos, 28130 Madrid, Spain
}

The genome of the Infectious Hematopoietic Necrosis Virus (IHNV), a salmonid Novirhabdovirus has been engineered to modify the gene order and to evaluate the impact on a possible attenuation of the virus in vitro and in vivo. By reverse genetics, eight recombinant IHNV (rIHNV), termed NxGy according to the respective position of the nucleoprotein $(\mathrm{N})$ and glycoprotein $(\mathrm{G})$ genes along the genome, have been recovered. All rIHNV have been fully characterized for their cytopathic effect, kinetics of replication, profile of viral gene transcription and their induced-immune response potential in fish. These rIHNV are stable through up to ten passages in cell culture. Following bath immersion administration of the various rIHNV to juvenile trout, some of the rIHNV were clearly attenuated (N2G3, N2G4, N3G4 and N4G1). Position of the N gene is one of the most critical features correlated to the level of viral attenuation. The recombinant virus N2G3 induced a strong antibody response in immunized fish and conferred $86 \%$ of protection against wild-type IHNV challenge in trout, thus representing a promising live-attenuated vaccine candidate.

KEYWORDS: reverse genetics, IHNV, trout, attenuation, vaccine

*Corresponding author. Tel.: +336889763 33

E-mail address: michel.bremont@jouy.inra.fr

${ }^{\$}$ Speaker: Stephane

E-mail address: stephane.biacchesi@jouy.inra.fr 